\title{
ON GALOIS SUBRINGS OF A FULL RING OF LINEAR TRANSFORMATIONS ${ }^{1}$
}

\section{HIRAM PALEY}

Although several authors, including Dieudonné [1], Hochschild [3], Nakayama [5], Rosenberg and Zelinsky [9], and Jacobson [4], have worked on the Galois theory of simple rings with chain conditions, much of their work is concerned with 1-1 correspondences between groups of automorphisms of a simple ring and Galois subrings. Very little, however, has been done towards classifying those subrings of a simple ring which are Galois. Jacobson [4] gives a condition due to Nakayama for a weakly Galois completely reducible subring to be Galois. In this paper we give a sufficient condition for a non-necessarily completely reducible weakly Galois subring to be Galois. Our result overlaps Nakayama's but does not properly contain his.

Throughout this paper we shall use the following notations: $\Delta$, a division ring finite dimensional over its center $\Gamma, \Gamma \neq \mathrm{GF}(2) ; M$, a finitely generated $\Delta$-module; $L=\operatorname{Hom}_{\Delta}(M, M) ; R$, a subring of $L$ containing $\Gamma$. We assume that $M$ as a module over any ring considered is a left-module.

Since $M$ is a finite dimensional vector space over $\Delta$, it is also a finite dimensional vector space over $\Gamma$. Thus $\operatorname{Hom}_{\Gamma}(M, M)$ can be regarded in the usual way as a finitely generated algebra over $\Gamma$. And since the rings $R, \Delta, \Gamma, L$, and $\Delta R$ are contained in $\operatorname{Hom}_{\Gamma}(M, M)$, they are all finite dimensional over $\Gamma$ and thus all these rings satisfy all the chain conditions and $M$ is a finitely generated module over each of them.

LEMMA $1 .^{2}$ If $\Delta$ is a division ring finite dimensional over its center $\Gamma$, if $R$ is any $\Gamma$-algebra and if $M$ is an $(R \otimes \Delta)$-module which is injective as an $R$-module, then $M$ is also injective as an $(R \otimes \Delta)$-module.

Proof. This follows immediately from [2, Proposition 2, p. 74], which asserts 1 . inj. $\operatorname{dim}_{R \otimes \Delta} M \leqq \operatorname{dim} \Delta+1$. inj. $\operatorname{dim}_{R} M$, because $\operatorname{dim} \Delta=0$ since $\Delta$ is separable over $\Gamma$ and 1 . inj. dim. is zero wherever the module is injective and conversely.

Presented to the Society, November 28, 1959; received by the editors September $12,1960$.

${ }_{1}^{1}$ Part of the research on this paper was done while the author was a National Science Foundation Pre-doctoral Fellow and the writing of this paper was supported in part by the Office of Naval Research.

2 The author wishes to thank the referee for his suggestions in general and in particular for calling the proof of this lemma to the author's attention. 
Lемма 2. If $R$ is a quasi-Frobenius subring of $\operatorname{Hom}_{\Delta}(M, M)$, $R \supseteq \Gamma$, then $\Delta R \cong\left(\right.$ isomorphic) $\Delta \otimes_{\Gamma} R$ and $\Delta R$ is quasi-Frobenius.

Proof. By $\left[4\right.$, Corollary 5.6, 1], $\Delta R \cong \Delta \otimes_{\Gamma} R$ if $R$ and $\Delta$ commute, if $\Delta$ is central simple and if no nonzero element of $\Delta$ annihilates $R$. These conditions are satisfied here. By $[7$, Theorem 14], the tensor product of quasi-Frobenius algebras of finite dimension is quasiFrobenius.

Leмма 3. If $R$ is quasi-Frobenius and if $R^{\prime}=\operatorname{Hom}_{R}(M, M)$ is generated over $\Delta$ by 1-1 $\Delta$-semi-linear transformations of $M$, then $R$ is Galois in L.

Proof. Let $S$ be the set of semi-linear transformations in $R^{\prime}$ with inverses. Clearly, $R^{\prime}=\Delta S$. Let $G$ be the group of inner automorphisms of $\operatorname{Hom}_{\Gamma}(M, M)$ produced by elements of $S$. The group $G$ induces a group of automorphisms on $L$ whose fixed ring is $F=\{x \mid x s=s x$ for all $s$ in $S\}=$ centralizer of $\Delta S=\operatorname{Hom}_{R^{\prime}}(M, M)$. Since $R$ is quasi-Frobenius, $\operatorname{Hom}_{R^{\prime}}(M, M)=R$ by the doublecentralizer theorem [8], so $F=R$ and $R$ is the fixed ring under the restriction of $G$ to $L$.

Theorem. If $R$ is quasi-Frobenius, if $M$ is an injective $R$-module, and if $R$ is weakly Galois in $L$, then $R$ is Galois in $L$.

Proof. By Lemma 3, it suffices to prove that $\operatorname{Hom}_{R}(M, M)$ is generated over $\Delta$ by 1-1 $\Delta$-semi-linear transformations of $M$.

Decompose $M$ into a direct sum $\sum^{\oplus} M_{i}$ of indecomposable $\Delta R$ modules. By the above lemmas, the $M_{i}$ are all injective.

CASE 1. $s$ is a semi-linear transformation which is zero on $\sum_{i>1}^{\oplus} M_{i}$ and has kernel zero on $M_{1}$. Since $s$ is semi-linear, it corresponds to some automorphism of $\Delta$ over $\Gamma$, which must be an inner automorphism because $\Delta$ is finite-dimensional and central simple over $\Gamma$. Thus there is a $\mu \neq 0$ in $\Delta$ such that for all $\delta$ in $\Delta, s \delta=\left(\mu^{-1} \delta \mu\right) s$. Thus, $\mu s$ commutes with $\Delta$ as well as with $R, \mu s$ has kernel zero on $M_{1}$ and so $\mu s$ gives a $\Delta R$-isomorphism of $M_{1}$ onto $\mu s\left(M_{1}\right)=s\left(M_{1}\right)$. This proves that $s\left(M_{1}\right)$ is indecomposable and injective over $\Delta R$. We decompose $M$ a second time: $M=s\left(M_{1}\right) \oplus \sum_{i>1}^{\oplus} M_{i}^{\prime}$. By Krull-Schmidt, we can find isomorphisms $\phi_{i}$ of $M_{i}$ onto $M_{i}^{\prime}(i=2, \cdots, n)$. Since $\Gamma \neq \mathrm{GF}(2)$, choose $\gamma \neq 0,1$ in $\Gamma$. Define $s_{1}$ to be $\gamma s$ on $M_{1}, \mu^{-1} \phi_{i}$ on $M_{i}(i>1)$ and define $s_{2}$ to be $(1-\gamma) s$ on $M_{1},-\mu^{-1} \phi_{i}$ on $M_{i}$, so that $s=s_{1}+s_{2}$ and each of $s_{1}, s_{2}$ is a semi-linear transformation with inverse.

CASE 2. $s=0$ on $\sum_{i>1}^{\oplus} M_{i}$ and $(\operatorname{ker} s) \cap M_{1} \neq 0$. Then $(\operatorname{ker} s) \cap M_{1}$ must contain the unique minimal submodule of $M_{1}$. Define $s_{1}=s+\mu^{-1}$ on $M_{1}$ ( $\mu$ as in Case 1) and zero on $\sum_{i>1}^{\oplus} M_{i}$, define $s_{2}=-\mu^{-1}$ on $M_{1}$ and zero on $\sum_{i>1}^{\oplus} M_{i}$. Then $s=s_{1}+s_{2}$ and $s_{1}$ and $s_{2}$ each belong to Case 1. 
CASE 3. $s=s_{1}+\cdots+s_{n}$, where $s_{j}$ coincides with $s$ on $M_{j}$ and is zero on $\sum_{i \neq j}^{\oplus} M_{i}$. By Case 2 (with 1 changed to $j$ ), $s_{j}$ is a sum of semi-linear transformations in $R^{\prime}$ with inverses. Thus so is $s$.

Since any $s$ in $\operatorname{Hom}_{R}(M, M)$ must belong to one of the above cases, the theorem is proved.

COROLlaRY. If $R$ and $R^{\prime}$ are quasi-Frobenius, $R^{\prime} \supseteq R \supseteq \Gamma, R^{\prime} \subseteq L$, if $M$ is injective as both an $R$-and $R^{\prime}$-module, and if $R$ is weakly Galois in $L$, then $R$ and $R^{\prime}$ are Galois in $L$.

Proof. $R$ is Galois by the Theorem. That $R^{\prime}$ is weakly Galois if $R$ is weakly Galois is well known (see, for example, [4, p. 149, proof of Fundamental Theorem ]), so $R^{\prime}$ also satisfies the hypotheses of the theorem.

REMARK 1. If $M$ is a direct sum of isomorphic indecomposable $R$ modules and if $R$ is quasi-Frobenius, then $M$ is an injective $R$-module.

Proof. $M$ contains as a direct summand the reduced regular representation of $R$. Thus, all the direct summands of $M$ are isomorphic to $R e, e$ a primitive idempotent in $R$. It is well known that $R e$ is injective.

REMARK 2. If $R$ is a principal ideal subring of $L$, then $R$ is quasiFrobenius. This follows immediately from [6, Theorem 2, p. 285 and Lemma 2, p. 286]. A special case is the ring $\Gamma[T]$ generated over $\Gamma$ by a single $\Delta$-linear transformation $T$ of $M$.

This paper formed part of a doctoral dissertation written under Professor Charles W. Curtis at the University of Wisconsin. The author wishes to express his deep appreciation to Professor Curtis for his many valuable suggestions.

\section{BIBLIOGRAPHY}

1. Jean Dieudonné, La théorie de Galois des anneaux simples et semi-simples, Comment. Math. Helv. vol. 21 (1948) pp. 154-184.

2. S. Eilenberg, A. Rosenberg, and D. Zelinsky, On the dimension of modules and algebras. VIII. Dimension of tensor products, Nagoya Math. J. vol. 12 (1957) pp. 71-93.

3. G. Hochschild, Automorphisms of simple algebras, Trans. Amer. Math. Soc. vol. 69 (1950) pp. 292-301.

4. N. Jacobson, Structure of Rings, Amer. Math. Soc. Colloquium Publications, vol. $37,1956$.

5. T. Nakayama, Galois theory of simple rings, Trans. Amer. Math. Soc. vol. 73 (1952) pp. 276-292.

6. - Note on uni-serial and generalized uni-serial rings, Proc. Imp. Acad. Japan vol. 16 (1940) pp. 285-289.

7. - On Frobeniusean algebras. II, Ann. of Math. vol. 42 (1941) pp. 1-21.

8. C. J. Nesbitt and R. M. Thrall, Some ring theorems with applications to modular representations, Ann. of Math. vol. 47 (1946) pp. 551-567.

9. A. Rosenberg and D. Zelinsky, Galois theory of continuous linear transformation rings, Trans. Amer. Math. Soc. vol. 79 (1955) pp. 429-452.

UNIVERSITY OF ILLINOIS 